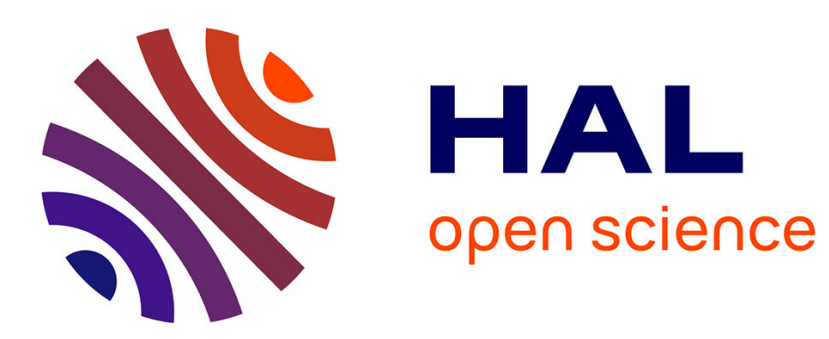

\title{
Using Pattern Structures for Analyzing Ontology-Based Annotations of Biomedical Data
}

\author{
Adrien Coulet, Florent Domenach, Mehdi Kaytoue, Amedeo Napoli
}

\section{To cite this version:}

Adrien Coulet, Florent Domenach, Mehdi Kaytoue, Amedeo Napoli. Using Pattern Structures for Analyzing Ontology-Based Annotations of Biomedical Data. International Conference on Formal Concept Analysis, May 2013, Dresden, Germany. hal-00880643

\section{HAL Id: hal-00880643 \\ https://hal.inria.fr/hal-00880643}

Submitted on 13 Dec 2013

HAL is a multi-disciplinary open access archive for the deposit and dissemination of scientific research documents, whether they are published or not. The documents may come from teaching and research institutions in France or abroad, or from public or private research centers.
L'archive ouverte pluridisciplinaire HAL, est destinée au dépôt et à la diffusion de documents scientifiques de niveau recherche, publiés ou non, émanant des établissements d'enseignement et de recherche français ou étrangers, des laboratoires publics ou privés. 


\title{
Using pattern structures for analyzing ontology-based annotations
}

\author{
Adrien Coulet ${ }^{1,2}$, Florent Domenach ${ }^{3}$, Mehdi Kaytoue $^{4}$, and Amedeo Napoli ${ }^{1,5}$ \\ 1 Inria, Villers-lès-Nancy, F-54600, France adrien.coulet, amedeo.napoli@loria.fr \\ 2 Université de Lorraine, LORIA, UMR 7503, Vandoeuvre-lès-Nancy, F-54506, France \\ ${ }^{3}$ Computer Science Department, University of Nicosia, 46 Makedonitissas Av., \\ P.O.Box 24005, 1700 Nicosia, Cyprus, domenach.f@unic.ac.cy \\ 4 Université de Lyon, CNRS, INSA-Lyon, LIRIS, UMR5205, F-69621, France \\ mkaytoue@liris.cnrs.fr \\ ${ }^{5}$ CNRS, LORIA, UMR 7503, Vandoeuvre-lès-Nancy, F-54506, France
}

\begin{abstract}
Annotating data with concepts of an ontology is a common practice in the biomedical domain. Resulting annotations (data-concept relationships) are useful for data integration whereas the background ontology can guide the analysis of integrated data. Formal Concept Analysis (FCA) allows to build from a binary context a concept lattice that can be used for data analysis purposes. However annotated biomedical data are not binary and a binarization procedure is required as a preprocessing, coming with classical problems, e.g. a trade-off between expressivity and the large number of induced binary attributes. Interestingly, pattern structures offer a general method for building a concept lattice from any set of objects associated with partially ordered descriptions. In this paper, we show how to instantiate this general framework when the space of descriptions is based on an ontology. We illustrate our approach with the analysis of biomedical annotations and we show its capabilities for knowledge discovery.
\end{abstract}

\section{Introduction}

Annotating data with concepts of an ontology is a common practice in the biomedical domain. Resulting annotations define links between data and concepts that are a support for data exchange, data integration and data analysis tasks [17. Annotations can be obtained in three main ways. In manual annotation, the specification of links between data and concepts is provided by human domain experts. Automated annotation consists in programs parsing data to provide such links. Semi-automated annotation is a combination of the two previous ways, where programs suggest links between data and concepts that are subsequently validated by domain experts [16. Here we are interested in the analysis of annotations of several data resources w.r.t. a "reference ontology". This allows a conjoint use of data from different biomedical domains, e.g., molecular biology and medicine. Consequently, the annotation process plays a major role in translational bioinformatics whose objective is to analyze molecular biomedical data and to discover correlations with clinical knowledge [6]. In this way, 
the search for hypotheses about molecular mechanisms underlying translational bioinformatics and the discovery of connexions between molecular data and clinical observations can be performed through the analysis of annotations (as links between biomedical data and ontological concepts).

Formal Concept Analysis (FCA) is a mathematical framework for data analysis and knowledge discovery [8]. As such, it can be used for analyzing annotations of biomedical data provided that some adaptations are made. Firstly, annotations are considered as pairs < document, set of concepts $>$ and thus cannot be directly represented within a binary context. Secondly, domain knowledge in the reference ontology is used for the annotation process and should also be taken into account as well in the analysis process. Thus annotations appear like complex data to be analyzed with FCA, requiring at least binarization.

A first solution is given by scaling, which relies on a transformation of nonbinary data into binary data. Several types of scaling are known in the FCA literature, e.g., nominal, ordinal, interordinal [8]. But it is also known that scaling leads to several problems such as arbitrary transformation of data, data loss and a potential binary attribute flooding, forbidding a comprehensive visualization of the results (see for example experiments and discussion in [10]).

Another solution is to use pattern structures that allows to directly analyze the complex data 7. In this setting, objects may have a complex description (i.e., non-binary) but the set of descriptions must be partially ordered within a semi-lattice of descriptions. Descriptions can be of many types, i.e., numerical intervals [11, set of attributes [7] or graphs [14. Pattern structures allow the application of standard FCA algorithms, e.g., for building the concept lattice, to a partially ordered set (a poset) of descriptions. The partial order on descriptions is defined thanks to a so-called similarity operator (also called meet) and an associated subsumption relation. The formalism of pattern structures was introduced in 7] and gained a lot of interest in the last years due to the need for data mining and knowledge discovery associated with the availability of large volumes of web data (i.e., complex data).

In this paper, we present a first approach to analyze annotations based on a reference ontology using the formalism of pattern structures. The present approach can be seen as a materialization of what is termed as structured attribute sets in [7. A first requirement for using pattern structures is to define descriptions of objects, then a similarity operation with its associated subsumption relation (thus a partial ordering on descriptions). In the present case, annotations are based on concepts of a reference ontology, which is itself based on two posets, a poset of concepts and a poset of relations (here only the poset of concepts will be taken into account). Accordingly, we propose in this paper an original adaptation of the formalism of pattern structures to annotations based on set of concepts from a reference ontology. Here, descriptions of objects are given by sets of concepts. Then, the ordering of concepts in the reference ontology is used to define an original similarity operator and the associated subsumption relation on descriptions. This is - to the best of our knowledge - the first attempt to analyze data annotations with a pattern structure. Moreover, this shows the potential 
of pattern structures as an effective formalism for dealing with real-world data and providing substantial results. Actually, the resulting concept lattice can be used for guiding the document annotation process, and especially for completing annotations that are given by an automatic annotation tool. In this case, annotations may be wrong or incomplete. The work of a domain expert for correcting and completing annotations can be very time consuming when large data are considered.

The paper is organized as follows. Section 2 recalls fundamental definitions used in the paper. Section 3 presents our adaptation of pattern structures to ontology-based annotations. It introduces also a concrete example about biomedical data for illustrating the approach. Section 4 details the similarity and subsumption operations on descriptions, while Section 5 provides a discussion about the analysis of annotations of biomedical data using our approach.

\section{Background definitions}

\subsection{Formal Concept Analysis}

We recall here the standard FCA notations and we refer readers to 8 for details and proofs. A formal context $(G, M, I)$ is defined as a set $G$ of objects, a set $M$ of attributes, and a binary relation $I \subseteq G \times M .(g, m) \in I$ means that "the object $g$ is related with the attribute $m$ through the relation $I$ ". Two derivation operators can be defined on sets of objects and sets of attributes as follows, $\forall A \subseteq G, B \subseteq M:$

$$
\begin{aligned}
& A^{\prime}=\{m \in M: \forall g \in A,(g, m) \in I\} \\
& B^{\prime}=\{g \in G: \forall m \in B,(g, m) \in I\}
\end{aligned}
$$

The two operators $(\cdot)^{\prime}$ define a Galois connection between the power set of objects $\mathcal{P}(G)$ and the power set of attributes $\mathcal{P}(M)$. A pair $(A, B), A \subseteq G, B \subseteq$ $M$, is a formal concept iff $A^{\prime}=B$ and $B^{\prime}=A$. A is called the extent and $\mathrm{B}$ the intent of the concept. The set of all formal concepts, ordered by inclusion of extents (or dually by inclusion of intents), i.e., $\left(A_{1}, B_{1}\right) \leq\left(A_{2}, B_{2}\right)$ iff $A_{1} \subseteq A_{2}$ (or dually $B_{2} \subseteq B_{1}$ ), forms a complete lattice [4, called concept lattice.

\subsection{Pattern structures}

A pattern structure can be understood as a generalization of a formal context to describe complex data 7): An object has a description lying in a semi-lattice where an "intersection" (or meet) is defined. This intersection allows to characterize the similarity between two descriptions, i.e. what they do have in common.

Formally, let $G$ be a set of objects, let $(\mathcal{D}, \sqcap)$ be a meet-semi-lattice of object descriptions and let $\delta: G \longrightarrow \mathcal{D}$ be a mapping associating each object with its description. $(G,(\mathcal{D}, \sqcap), \delta)$ is called a pattern structure. Elements of $\mathcal{D}$ are called descriptions or patterns and are ordered by a subsumption relation $\subseteq$ such as 
$\forall c, d \in \mathcal{D}, c \sqsubseteq d \Longleftrightarrow c \sqcap d=c$. A pattern structure $(G,(\mathcal{D}, \sqcap), \delta)$ gives rise to two derivation operators denoted by $(\cdot)^{\square}$ :

$$
\begin{array}{cc}
A^{\square}=\prod_{g \in A} \delta(g) & \text { for } A \subseteq G \\
d^{\square}=\{g \in G \mid d \sqsubseteq \delta(g)\} & \text { for } d \in(\mathcal{D}, \sqcap) .
\end{array}
$$

These operators form a Galois connection between the power set of objects $\mathcal{P}(G)$ and $(\mathcal{D}, \sqcap)$. Pattern concepts of $(G,(\mathcal{D}, \sqcap), \delta)$ are pairs of the form $(A, d), A \subseteq G$, $d \in(\mathcal{D}, \sqcap)$, such that $A^{\square}=d$ and $A=d^{\square}$. For a pattern concept $(A, d), d$ is the pattern intent and is the common description to all objects in $A$, the pattern extent. When partially ordered by $\left(A_{1}, d_{1}\right) \leq\left(A_{2}, d_{2}\right) \Leftrightarrow A_{1} \subseteq A_{2} \quad\left(\Leftrightarrow d_{2} \subseteq d_{1}\right)$, the set of all concepts forms a complete lattice called pattern concept lattice. The operator $(\cdot)^{\square \square}$ is a closure operator and pattern intents are closed patterns. Pattern structure have been applied to numerical intervals [1] and to graphs 14.

\section{$2.3 \quad \mathcal{E} \mathcal{L}$ ontologies}

Ontologies that are considered in this work are DL ontologies, i.e. are based on a set of concepts and relations represented with a Description Logic (DL) 2].

The $\mathcal{E} \mathcal{L}$ DL allows for conjunction $(\wedge)$ and existential restriction $(\exists r . c)$ in definitions of concepts [1]. This simple DL is sufficient for our purpose, together with transitive roles and general concept inclusion axioms i.e., axioms of the form $C \leqslant D$ where $C, D$ can be either atomic or defined concepts. Moreover, the least common subsumer (lcs) of two concepts in $\mathcal{E} \mathcal{L}$ always exists and can be computed in polynomial time, provided that their is no cycle in concept definitions, i.e., the definition of a concept $c_{i}$ does not include $c_{i}$ itself [3].

In order to avoid any confusion and to make a clear distinction between the DL formalism and the pattern structure formalism, we use the classical logical notation ${ }^{6}$ for the $\mathcal{E} \mathcal{L}$ DL, thus $\wedge$ for conjunction and $\leqslant$ for subsumption, while we keep $\sqcap$ for the similarity operator and $ᄃ$ for the subsumption relation in pattern structures.

In the following, we consider a reference ontology denoted by $\mathcal{O}$ based on the $\mathcal{E} \mathcal{L}$ DL. $\mathcal{O}$ is composed of:

- $C(\mathcal{O})$ denotes a set of concepts, and $R(\mathcal{O})$ denotes a set of binary relations $\$$.

- concepts $c_{i}$ in $C(\mathcal{O})$ are partially ordered thanks to a subsumption relation $\leqslant$, where $c_{1} \leqslant c_{2}$ means that concept $c_{1}$ is a sub-concept of $c_{2}$ and that every instance of $c_{1}$ is an instance of $c_{2}$,

$-A$ is a set of axioms involving concepts and relations.

\footnotetext{
${ }^{6}$ But not classical in DL.

7 To avoid confusion, we will use the terms concept for DL ontologies and formal concepts or pattern concepts for FCA.
} 


\section{Problem statement}

\subsection{The UMLS Semantic Network and semantic types}

The UMLS (Unified Medical Language System) is composed of two main components: a set of ontologies of various biomedical domains (such as SNOMED CT, ICD-10, MeSH) and the UMLS Semantic Network [5]. For a sake of simplicity, we illustrate our study with annotations of a single data resource, DrugBank 8 [12, made with a single ontology of the UMLS, the NCI (National Cancer Institute) Thesaurus [18.

The UMLS Semantic Network is a set of broad subject categories, or semantic types, that is used as a high level categorization of concepts of UMLS ontologies [15. An overview of the 133 semantic types is available at http://www.nlm. nih.gov/research/umls/META3_current_semantic_types.html. The Semantic Network organizes semantic types as a simple hierarchy, i.e., a tree denoted hereafter as $\mathcal{S} \mathcal{T}_{\text {hierarchy. }}$. For example, some semantic types are broader than others such as "Organism" that is more general than "Human" or "Anatomical Structure" more general than "Tissue".

Every concept of a UMLS ontology is mapped with one or more semantic types. In addition, the hierarchy of the Semantic Network $\mathcal{S} \mathcal{T}_{\text {hierarchy }}$ can be used to map a concepts $c_{1}$ with the set of semantic types that are ancestors of the semantic types of $c_{1}$. For example, if the concept $c_{1}$ has for semantic type "Disease or Syndrome", it can be mapped with "Pathologic Function" and "Biologic Function" too (as the laters are ancestors of the former in $\mathcal{S} \mathcal{T}_{\text {hierarchy }}$ ). Accordingly, we are using the hierarchy $\mathcal{S} \mathcal{T}_{\text {hierarchy }}$ to dispose of the full set of semantic types that can be mapped to each concept. Figure 1 illustrates the mappings of some concepts of the NCI Thesaurus with their semantic types.

In our approach, a selection of semantic types chosen by the analyst will be used as upper level categories for concepts annotating biomedical documents.

\subsection{Building a pattern structure for biomedical annotations}

In this work, we are interested in the discovery of associations between semantic categories (i.e., semantic types) of concepts annotating biomedical documents. This knowledge discovery method should take into account domain knowledge, i.e., the NCI Thesaurus, and semantic types. For example, an expert may be interested in a drug-disease association, e.g., Antibiotic-Inflammation, checking whether the association is frequent and searching for a potential associated molecular mechanism.

For analyzing annotations it may be worth to distinguish concepts thanks to domains of interests (kinds of points of view). For example, a domain expert may group concepts according to their membership to distinct ontologies to separate concepts from an ontology on disease and concepts from an ontology on drugs. Accordingly, we consider in this work that the domain expert defines a "scale"

\footnotetext{
${ }_{8}^{8}$ Publicly available at http://www.drugbank.ca/
} 


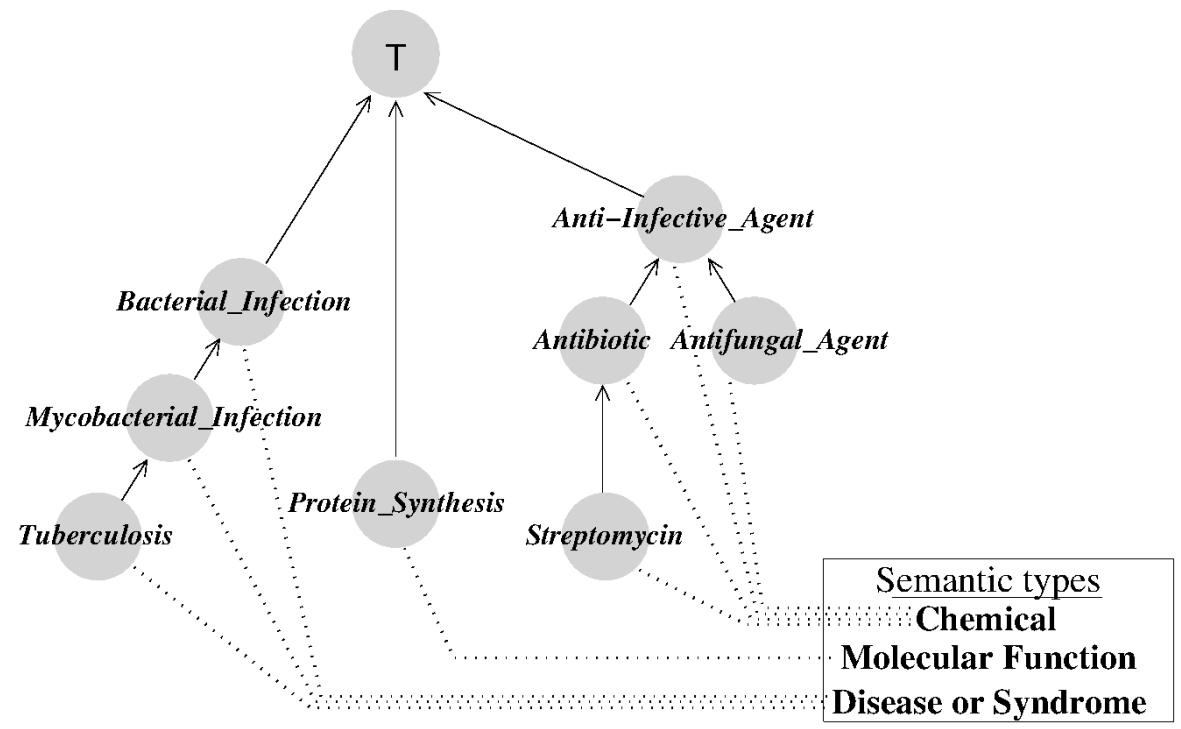

Fig. 1. Detail of the NCI Thesaurus with associated semantic types from the UMLS. Nodes are concepts of the ontology, arrows represent subsumption relationships $(\leqslant)$. Doted lines map each concept with its semantic type as defined in the UMLS Semantic Network.

$\mathcal{S T}=\left\{\mathrm{ST}_{1}, \mathrm{ST}_{2}, \ldots, \mathrm{ST}_{k}\right\}$ supporting the document annotations, where each $\mathrm{ST}_{i}$ is a semantic type. Then a biomedical document will be annotated w.r.t. the ST scale. More precisely, given a biomedical document $g$, the annotation of $g$ w.r.t. the reference ontology $\mathcal{O}$ and the $\mathrm{ST}$ scale is a pair $\left(g,\left\langle\mathrm{ST}_{1}, \mathrm{ST}_{2}, \ldots, \mathrm{ST}_{k}\right\rangle\right)$ where $\mathrm{ST}_{i}$ is the set of concepts annotating $g$ for the dimension $i$ in the scale $\mathcal{S T}$.

For example, let us consider the document DB01082 (gathering data about Streptomycin) in the DrugBank database. Figure 2 shows this document and an annotation with three concepts of the NCI Thesaurus (here the reference ontology $\mathcal{O}$ ). Moreover, let us consider the $\mathcal{S} \mathcal{T}$ scale as $\mathcal{S} \mathcal{T}=\{$ "Disease or Syndrome", "Bacterium", "Molecular Function", "Chemical" $\}$. Then the annotation of DB01082 can be read as:

(DB01082, $\langle\{$ Tuberculosis $\},\{\},\{$ Protein_Synthesis $\},\{$ Streptomycin $\}\rangle)$

Now we have everything for defining the pattern structure $(G,(\mathcal{D}, \sqcap), \delta)$ for analyzing annotations of biomedical documents:

$-G=\left\{g_{1}, g_{2}, \ldots, g_{n}\right\}$ is a set of annotated biomedical documents;

$-\mathcal{O}$ is the reference ontology, i.e., the NCI Thesaurus, and $C(\mathcal{O})$ is the set of concepts of $\mathcal{O}$;

$-\mathcal{S T}=\left\{\mathrm{ST}_{1}, \mathrm{ST}_{2}, \ldots, \mathrm{ST}_{k}\right\}$ is the set of semantic types of the UMLS Semantic Network that defines the scale $\mathcal{S T}$ and the dimensions of the annotation vector; 


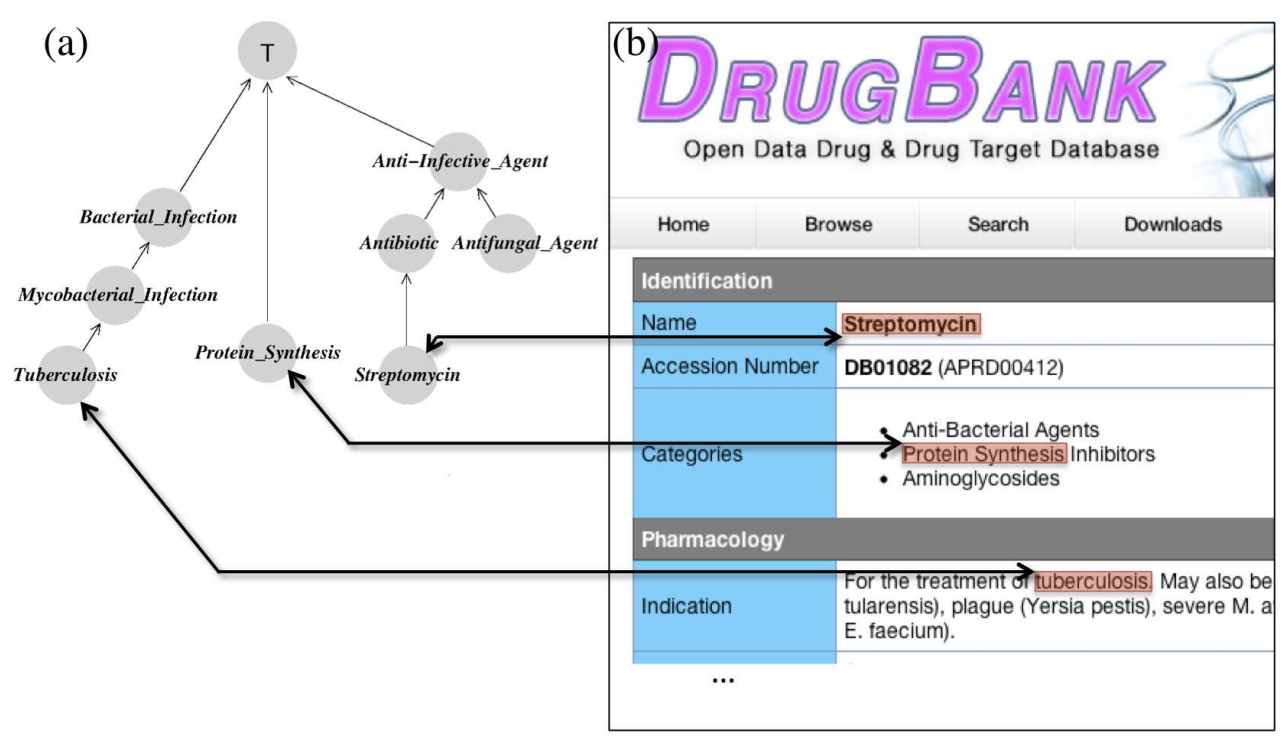

Fig. 2. (a) The left part of the Figure shows the NCI Thesaurus ontology; (b) the right part is an excerpt of the document DB01082 of DrugBank related to the Streptomycin drug. Bold arrows connecting (a) and (b) represent the annotation of DB01082.

$\left.-\mathcal{D}=\mathcal{P}\left(\mathrm{ST}_{1}\right) \times \mathcal{P}\left(\mathrm{ST}_{2}\right) \times \ldots \times \mathcal{P}\left(\mathrm{ST}_{k}\right)\right)$ where $\left.\mathcal{P}\left(\mathrm{ST}_{i}\right)\right)$ is the power set of the set of concepts of semantic type $\mathrm{ST}_{i}$. As a product of complete lattices, $\mathcal{D}$ is also a complete lattice (and thus a semi-lattice). Elements of $\mathcal{D}$ are named hereafter ontological patterns;

$-\delta: G \rightarrow \mathcal{D}$ is a mapping associating a document $g_{i} \in G$ with a description in $\mathcal{D}$ or more precisely a vector in $\mathcal{D}$,

$$
\delta\left(g_{i}\right)=\left(g_{i},\left\langle\mathrm{ST}_{1}\left(g_{i}\right), \mathrm{ST}_{2}\left(g_{i}\right), \ldots, \mathrm{ST}_{k}\left(g_{i}\right)\right\rangle\right)
$$

where $\mathrm{ST}_{j}\left(g_{i}\right)$ is the set of concepts of semantic type $\mathrm{ST}_{j}$ annoting $g_{i}$.

Table 1 gives an example of this pattern structure. The fourth line of the table shows the annotation of the document DB01082 (about Streptomycin). The different columns are filled with the concepts annotating DB01082 w.r.t. the semantic type provided in the header of each column.

Now, it remains to define the similarity operation $\square$ between two descriptions $\delta\left(g_{1}\right)$ and $\delta\left(g_{2}\right)$ :

$$
\begin{gathered}
\delta\left(g_{1}\right)=\left(g_{1},\left\langle\mathrm{ST}_{1}\left(g_{1}\right), \mathrm{ST}_{2}\left(g_{1}\right), \ldots, \mathrm{ST}_{k}\left(g_{1}\right)\right\rangle\right) \\
\delta\left(g_{2}\right)=\left(g_{2},\left\langle\mathrm{ST}_{1}\left(g_{2}\right), \mathrm{ST}_{2}\left(g_{2}\right), \ldots, \mathrm{ST}_{k}\left(g_{2}\right)\right\rangle\right) \\
\delta\left(g_{1}\right) \sqcap \delta\left(g_{2}\right)=\left\langle\operatorname{ST}_{1}\left(g_{1}\right) \sqcap \operatorname{ST}_{1}\left(g_{2}\right), \mathrm{ST}_{1}\left(g_{1}\right) \sqcap \mathrm{ST}_{1}\left(g_{2}\right), \ldots, \mathrm{ST}_{k}\left(g_{1}\right) \sqcap \mathrm{ST}_{k}\left(g_{2}\right)\right\rangle
\end{gathered}
$$

where $\mathrm{ST}_{1}\left(g_{1}\right) \sqcap \mathrm{ST}_{1}\left(g_{2}\right)$ is the convex hull in $\mathcal{O}$ of all concepts in $\operatorname{ST}_{1}\left(g_{1}\right)$ and $\mathrm{ST}_{2}\left(g_{2}\right)$. The definition of the convex hull is made precise in the next section. 
Table 1. A context where objects are DrugBank documents and attributes are semantic types. Each document is annotated with a set of concepts of the NCI Thesaurus (our reference ontology) having distinct semantic types. The document DB01082 of DrugBank (on the fourth line) is annotated with three concepts, including the concept Tuberculosis of semantic type "Disease or Syndrome".

\begin{tabular}{|c|c|c|c|c|}
\hline $\mathrm{ST}^{\mathcal{S T}}$ & $\begin{array}{l}\text { Disease or } \\
\text { Syndrome }\end{array}$ & Bacterium & $\begin{array}{l}\text { Molecular } \\
\text { Function }\end{array}$ & Chemical \\
\hline Drug1 & $\begin{array}{c}\text { \{Tuberculosis } \\
\text { Bacterial_Infection }\}\end{array}$ & \{\} & $\{$ Protein_Synthesis $\}$ & $\begin{array}{c}\{\text { Antibiotic } \\
\text { Antifungal_Agent }\}\end{array}$ \\
\hline Drug2 & $\{$ Bacterial_Infection $\}$ & \{\} & $\{$ Protein_Synthesis $\}$ & \{\} \\
\hline Drug3 & $\begin{array}{c}\{\text { Tuberculosis } \\
\text { Bacterial_Infection }\}\end{array}$ & \{\} & \{\} & $\{$ Anti-Infective_Agent $\}$ \\
\hline DB01082 & $\{$ Tuberculosis $\}$ & \{\} & $\{$ Protein_Synthesis $\}$ & $\{$ Streptomycin $\}$ \\
\hline Drug5 & $\begin{array}{c}\{\text { Tuberculosis } \\
\text { Bacterial_Infection }\}\end{array}$ & \{\} & \{\} & $\begin{array}{c}\{\text { Antibiotic, } \\
\text { Antifungal_Agent }\}\end{array}$ \\
\hline
\end{tabular}

\subsection{Similarity between descriptions}

Given an ontology $\mathcal{O}$, and two concepts $c_{1}$ and $c_{2}$, the least common subsumer, denoted by $\operatorname{lcs}\left(c_{1}, c_{2}\right)$, is the most specific concept subsuming both $c_{1}$ and $c_{2}$ w.r.t. the ontology $\mathcal{O}$. Here $\mathcal{O}$ is an $\mathcal{E} \mathcal{L}$ ontology where no cycle appears in concepts definitions. Thus the lcs of two concepts of $\mathcal{O}$ always exists [3. More generally, the lcs operation can be defined (recursively) for a set of concepts $C_{n}=\left\{c_{1}, c_{2}, \ldots, c_{n}\right\}$ as follows:

$$
\forall n \in \mathbb{N}, \operatorname{lcs}\left(C_{n}\right)=\operatorname{lcs}\left(\operatorname{lcs}\left(C_{n-1}\right), c_{n}\right)
$$

For example, the lcs of Streptomycin and Antifungal_Agent is AntiInfective_Agent (see Figure 2).

The lcs itself could be used to define a simple similarity operation between two descriptions. One potential application here is to use the concept lattice based on ontological patterns to complete annotations associated with biomedical documents. In this way, taking into account the convex hull of a set of concepts allows to consider available concepts in the ontology which are linked to the initial set of concepts of the annotation. Moreover, if one concept has been missed by the annotation process and is available in the ontology, it will be potentially retrieved through the computation of the convex hull of the initial set of concepts.

Now, we define the convex hull of two concepts $c_{1}$ and $c_{2}$, denoted by $\operatorname{CVx}\left(c_{1}, c_{2}\right)$, as the set of concepts $\left\{x_{1}, x_{2}, \ldots, x_{n}\right\}$ verifying:

$-x_{i} \leqslant \operatorname{lcs}\left(c_{1}, c_{2}\right)$, and

- either $\left\{\begin{array}{l}x_{i} \geqslant c_{1} \text { and } x_{i} \wedge c_{1} \equiv c_{1} \text { or } \\ x_{i} \geqslant c_{2} \text { and } x_{i} \wedge c_{2} \equiv c_{2}\end{array}\right.$

$-x_{i}$ 丰 
For example, $\operatorname{CVx}($ Streptomycin, Antifungal_Agent $)=\{$ Anti-Infective_Agent, Antibiotic, Antifungal_ Agent, Streptomycin\}.

As for the lcs operation, the convex hull operation can be generalized (recursively) to a set of concepts $C_{p}=\left\{c_{1}, c_{2}, \ldots, c_{p}\right\}$ :

$$
\forall p \in \mathbb{N}, \operatorname{CVx}\left(C_{p}\right)=\operatorname{CVx}\left(\operatorname{CVx}\left(C_{p-1}\right), c_{p}\right)
$$

The meet operation on descriptions applies on two vectors with the same dimensions and returns a vector where the components are filled with the convex hull of the two initial sets of concepts. Formally we have:

$$
\begin{aligned}
& \delta\left(g_{1}\right)=\left(g_{1},\left\langle\mathrm{ST}_{1}\left(g_{1}\right), \mathrm{ST}_{2}\left(g_{1}\right), \ldots, \mathrm{ST}_{k}\left(g_{1}\right)\right\rangle\right) \\
& \delta\left(g_{2}\right)=\left(g_{2},\left\langle\mathrm{ST}_{1}\left(g_{2}\right), \mathrm{ST}_{2}\left(g_{2}\right), \ldots, \mathrm{ST}_{k}\left(g_{2}\right)\right\rangle\right) \\
& \delta\left(g_{1}\right) \sqcap \delta\left(g_{2}\right)=\left\langle\mathrm{ST}_{1}\left(g_{1}\right) \sqcap \mathrm{ST}_{1}\left(g_{2}\right), \mathrm{ST}_{2}\left(g_{1}\right) \sqcap \mathrm{ST}_{2}\left(g_{2}\right), \ldots, \mathrm{ST}_{k}\left(g_{1}\right) \sqcap \mathrm{ST}_{k}\left(g_{2}\right)\right\rangle \\
& \operatorname{ST}_{i}\left(g_{1}\right) \sqcap \operatorname{ST}_{i}\left(g_{2}\right)=\operatorname{CVX}\left(\operatorname{ST}_{i}\left(g_{1}\right) \cup \operatorname{ST}_{i}\left(g_{2}\right)\right) .
\end{aligned}
$$

The convex hull on the union of two sets of concepts is similar to the convex hull on a set of concepts as defined above.

It can be noticed that the definition of the meet operator on concepts can be likened to the the definition of the meet operator for numerical intervals as the convex hull of two intervals (see for example [11]). Moreover, similarly as for intervals we have the following property:

$$
\delta\left(g_{1}\right) \sqcap \delta\left(g_{2}\right)=\delta\left(g_{1}\right) \text { iff } \delta\left(g_{1}\right) \sqsubseteq \delta\left(g_{2}\right)
$$

As an illustration let us consider the two objects "Drug1" and "DB01082" and their descriptions $\delta($ Drug1) and $\delta(\mathrm{DB} 01082)$ given in the Table 1 . Their meet is

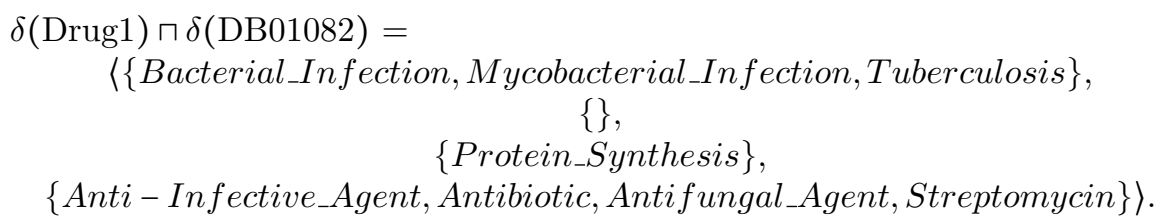

The meet semi-lattice of pattern elements (indeed of convex hulls) defined by the meet operation is given in Figure 3. This semi-lattice is associated with the context of Table 1 and the order defined by the NCI Thesaurus given in Figure 2.

Dually, it is also possible to define a join operation on descriptions, making $(\mathcal{D}, \sqcap, \sqcup)$ a complete lattice. This operation is not necessary for the definition of pattern structures but exists in our case because of the property of $\mathcal{D}$, the space of descriptions. The join of two descriptions $\delta\left(g_{1}\right)$ and $\delta\left(g_{2}\right)$ is defined as follows:

$$
\delta\left(g_{1}\right) \sqcup \delta\left(g_{2}\right)=\left\langle\mathrm{ST}_{1}\left(g_{1}\right) \sqcup \mathrm{ST}_{1}\left(g_{2}\right), \mathrm{ST}_{2}\left(g_{1}\right) \sqcup \mathrm{ST}_{2}\left(g_{2}\right), \ldots, \mathrm{ST}_{k}\left(g_{1}\right) \sqcup \mathrm{ST}_{k}\left(g_{2}\right)\right\rangle
$$




\section{$<$ Bacterial_Infection, \\ Mycobacterial_Infection,Tuberculosis\} \\ \{\} \{Protein_Synthesis\} \\ \{Aminoglycoside_Antibiotic,}

Anti-Infective_Agent,Antibiotic,Antifungal_Agent,Streptomycin\}>

$<$ Bacteriał_Infection,

Mycobacterial_Infection,Tuberculosis\}

\{\} \{Protein_Synthesis\}

\{Anti-Infective_Agent, Antibiotic, Antifungal_Agent\}>
$<$ Bacterial_Infection, Mycobacterial_Infection,

Tuberculosis\}

\{\} \{\}

\{Anti-Infective_Agent, Antibiotic,Antifungal_Agent $\}$

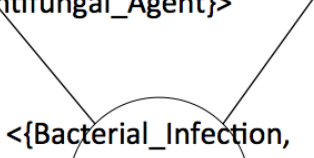

Mycobacterial_Infection,Tuberculosis\}

\{\} \{\}

\{Anti-Infective_Agent\}>
$<$ Bacterial_Infection,

Mycobacterial_Infection,

Tuberculosis\}

\{\} \{Protein_Synthesis\}

\{Anti-Infective_Agent\}>

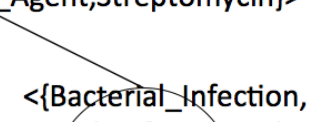

Mycobacterial_Infection,Tuberculosis\} \{\} \{Protein_Synthesis\}

\{Amingglycoside_Antibiotic,

Anti-Infective_Agent,Antibiotic,Streptomycin\}>

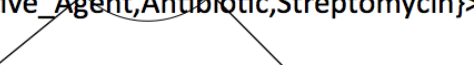

$<$ Bacterial_Infection, Mycobacterial_Infection, Tuberculosis\}

\{\} \{Protein_Synthesis\} \{Streptomycin\}>

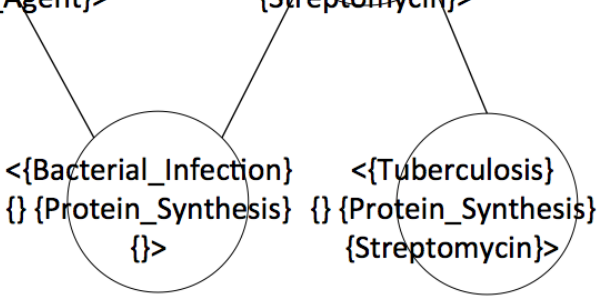

Fig. 3. The meet semi-lattice of convex hulls associated with the context represented in Table 1 and the NCI Thesaurus 
where

$$
\mathrm{ST}_{i}\left(g_{1}\right) \sqcup \mathrm{ST}_{i}\left(g_{2}\right)=\operatorname{CVX}\left(\mathrm{ST}_{i}\left(g_{1}\right)\right) \cap \operatorname{CVx}\left(\mathrm{ST}_{i}\left(g_{2}\right)\right) .
$$

Actually, the result of the join operation is the set of common concepts in the two convex hulls of $\mathrm{ST}_{i}\left(g_{1}\right)$ and $\mathrm{ST}_{i}\left(g_{1}\right)$.

For example, the join of the descriptions of "Drug1" and "DB01082" is:

$\delta($ Drug1 $) \sqcup \delta($ DB01082 $)=\langle\{$ Tuberculosis $\},\{\},\{$ Protein_Synthesis $\},\{\}\rangle$.

The intersection of two convex hulls may be empty as shown in the above example. However, it can be noticed that even if $\delta\left(g_{1}\right)$ and $\delta\left(g_{2}\right)$ may have no element in common, they can still have a join as illustrates the following example. Suppose that we have only one dimension and let us consider the reference ontology in Figure 2

$$
\begin{gathered}
\delta\left(g_{1}\right)=\langle\{\text { Bacterial_Infection, Tuberculosis }\}\rangle \\
\delta\left(g_{2}\right)=\langle\{\text { Mycobacterial_Infection }\}\rangle .
\end{gathered}
$$

Actually, the results of the meet and join operations on these two descriptions are:

$\delta\left(g_{1}\right) \sqcap \delta\left(g_{2}\right)=\langle\{$ Bacterial_Infection, Mycobacterial_Infection, Tuberculosis $\}\rangle$

and

$$
\delta\left(g_{1}\right) \sqcup \delta\left(g_{2}\right)=\langle\{\text { Mycobacterial_Infection }\}\rangle .
$$

In addition, we remark that we do not have $\delta\left(g_{1}\right) \sqcap \delta\left(g_{2}\right)=\delta\left(g_{1}\right)$ as $\delta\left(g_{1}\right)$ is not a convex hull and thus we do not have either $\delta\left(g_{1}\right) \subseteq \delta\left(g_{2}\right)$.

\subsection{Computing Pattern Structures with CloseByOne}

In FCA, an efficient way of computing closed formal concepts that are the basic bricks of concept lattices is the algorithm CloseByOne 13. To adapt CloseByOne to the general case of pattern structures, one has to replace the original Galois connexion, usually denoted $(\cdot)^{\prime}$, with the derivation operator denoted $(\cdot)^{\square}$. Below, we give the basic pseudo-code of the algorithm CloseByOne (Algorithms 1 and 2 for computing ontological patterns. In addition to the new derivation operator, one must replace the intersection operation on descriptions $(\cap)$ with the meet operation on patterns $(\sqcap$, line 5 of Algorithm 2 ) that is adapted to the nature of the patterns.

A simple implementation of Algorithms 1 and 2 is proposed at github.com/ coulet/OntologyPatternIcfca/

\section{Analyzing annotations of biomedical data}

We illustrate our approach with the analysis of annotations of DrugBank documents with the ontology named the NCI Thesaurus. These annotations are provided by the NCBO (National Center for Biomedical Ontology) Resource Index presented hereafter. 

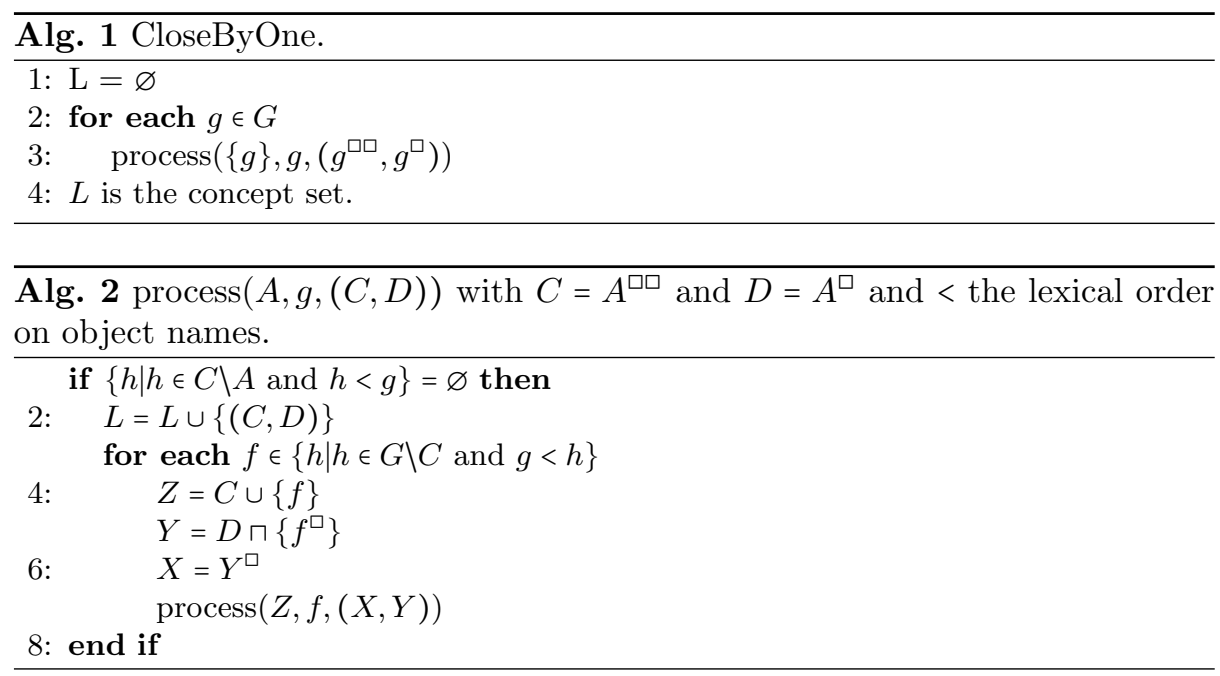

\subsection{A repository of annotations: The NCBO Resource Index}

The NCBO Resource Index is a repository of annotations automatically populated by a Natural Language Processing tool [9. This tool parses the textual content of several biomedical databases (e.g., DrugBank, OMIM, ClinicalTrial.gov) searching for occurrences of terms refering to concepts of ontologies. When a concept $c_{i}$ is found in a document $g_{i}$, an annotation i.e., a pair $\left(g_{i}, c_{i}\right)$, is created and stored. On December 18th, 2012, the NCBO Resource Index was containing annotations for 34 databases with concepts of 280 ontologies of the BioPortal 19. The Resource Index can be queried either by a Web user interfact ${ }^{9}$ or by a REST Web servict 10 . We used the second to build sets of annotations.

\subsection{DrugBank annotations with the NCI Thesaurus}

DrugBank is a publicly available database that contains data about drugs, their indications and their molecular targets. The database is organized into documents, or entries, every document gathering data about one drug. Data in DrugBank are for the main part made of texts is natural language. Figure 2 (b) presents the document of DrugBank that concerns Streptomycin.

As described above, the annotations we used to illustrate our approach are annotations of the DrugBank documents using concepts of the NCI Thesaurus. The NCI Thesaurus is a broad domain ontology and consequently its annotations may concern either clinics and molecular biology data that can be conjointly explored in translational bioinformatics. Moreover, the NCI Thesaurus is an $\mathcal{E} \mathcal{L}$ ontology that does not contain cyclic concept definition. Thus a lcs always exists

9 Available at http://bioportal.bioontology.org/resource_index

10 Documented at http://www.bioontology.org/wiki/index.php/Resource_Index_ REST_Web_Service_User_Guide 


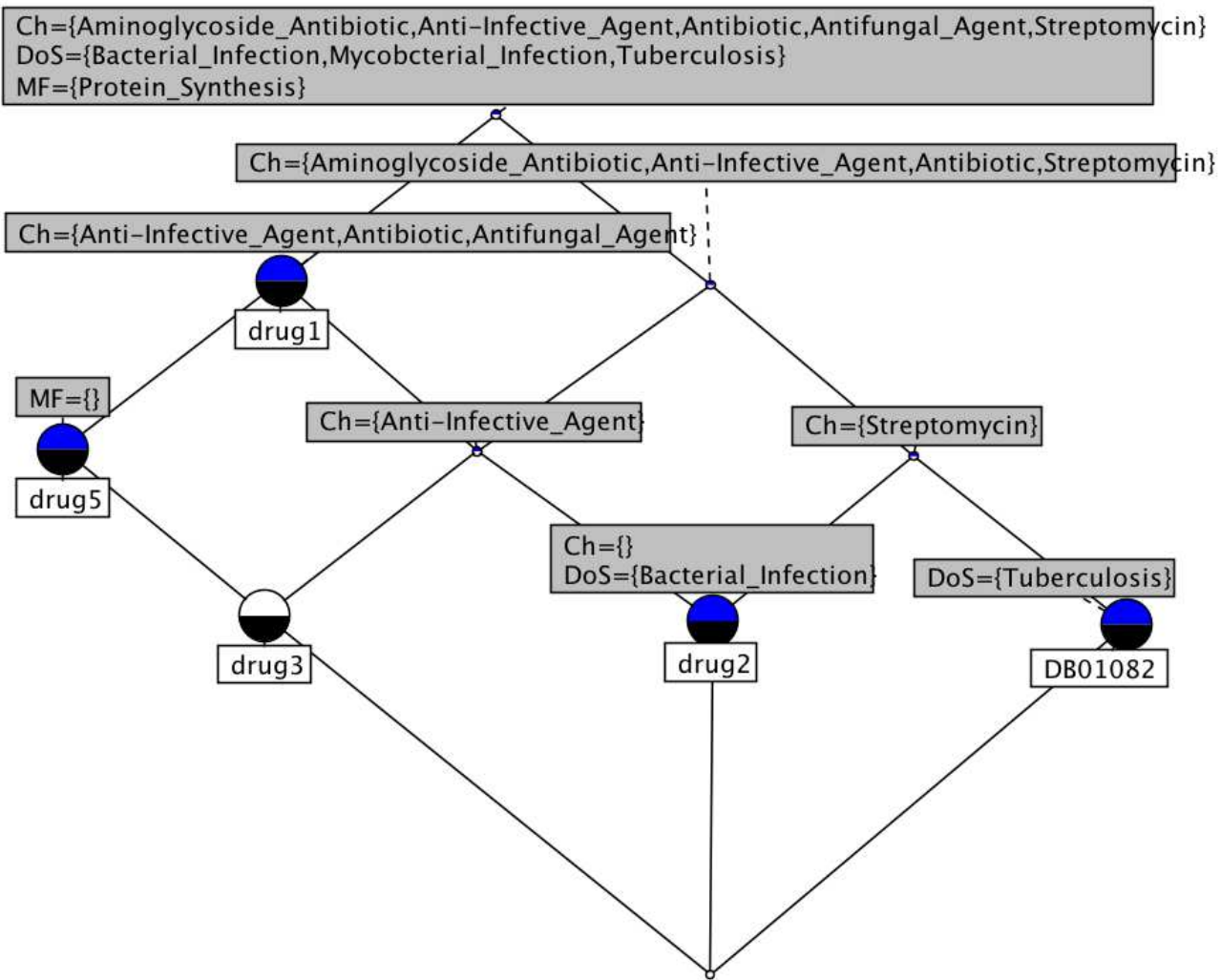

Fig. 4. The concept lattice corresponding to the pattern structure based on the context in Table 1 and on the NCI Thesaurus. The top concept has the intent with the larger descriptions and consequently its extent includes all the documents (objects). Traversing the lattice downward, the concepts present more specialized extents (with less objects) and more general intents w.r.t. the subsumption relation on ontological patterns. "Ch", "DoS" and "MF" are respectively abbreviations for the semantic types "Chemical", "Disease or Syndrome" and "Molecular Function").

and its processing is tractable. We used the version 12.04 of the NCI Thesaurus encoded in OWL and available on the NCBO Bioporta ${ }^{11}$

\subsection{Interpretation}

We propose in Table 1 a context including annotations of five DrugBank documents based on concepts to the NCI Thesaurus. Concepts may have four distinct semantic types (the cardinality of $\mathcal{S T}$ is four): "Disease or Syndrome", "Bacterium", "Molecular Function" and "Chemical". The meet-semi-lattice of

$\overline{11}$ NCI Thesaurus 12.04: bioportal.bioontology.org/ontologies/1032 
patterns associated with such annotations is depicted in Figure 3 and the corresponding concept lattice is given in Figure 4. Both sets of formal concepts in the semi-lattice and in the concept lattice have been obtained thanks to the implementation of CloseByOne that we adapted to ontological patterns (see subsection 3.4.

Now we propose an analysis of the resulting concept lattice shown in Figure 4 . Consider that one of our objectives is to repair and complete the annotations associated with biomedical documents. The top formal concept in the lattice has the "largest extent", i.e., the set of all the objects, and the "smallest intent", actually the largest convex hull for the annotations.

Let us consider the two formal concepts in the upper left part of the concept lattice, the first called $c_{\# 15}$ has an extent containing "drug1" and "drug5" and the second called $c_{\# 5}$ has an extent containing only "drug5". The "Chemical" semantic type (abbreviated "Ch" in Figure 4 of both concepts is AntiInfective_Agent, Antibiotic, Antifungal_Agent $\}$. The "Disease or Syndrome" dimension ("DoS") in both concepts is \{Bacterial_Infection, Mycobacterial_ Infection, Tuberculosis $\}$ as in the top concept. However, the "Molecular Function" dimension ("MF") is the same for the top concept and $c_{\# 15}$; i.e., \{Protein Synthesis\}, while it is redefined and empty in $c_{\# 5}$. We propose the following interpretation:

- The value of "Chemical" in both $c_{\# 15}$ and $c_{\# 5}$ is completed (as a convex hull) and is the correct annotation to be associated to document "drug1" and "drug5" for the "Chemical" dimension. This shows how the final concept lattice based on the ontological pattern structure can effectively complete the original annotation process (especially when this process is automated).

- The same remark applies to the "Disease or Syndrome" dimension, which is also completed (as a convex hull). The concept lattice provides once again the complete annotation for both concepts $c_{\# 15}$ and $c_{\# 5}$.

Thus, even on this small and toy example, it is possible to understand and verify the usefulness and potential of the approach: the resulting concept lattice yielded by the ontological pattern structure provides the means for completing the initial annotations in a way that respects the reference ontology.

Finally, we experimented the pattern approach on a larger real-world context. We selected 25 drugs of DrugBank out of 173 drugs returned by the query "antibiotic" and we retain the annotations provided by the NCBO Resource Index associated with 4 distinct semantic types. After 4.4 hours, we obtained 204, 801 closed concepts on a computer with two Intel Core 2 Extreme X7900 CPUs and $4 \mathrm{GiB}$ of memory. The resulting concept lattice is rather large and the analysis of formal concepts with a domain expert is in progress. We think that the results of the analysis will be in accordance with the analysis presented just above for the toy example. 


\section{Conclusion and Perspectives}

Pattern structures provide an original and efficient approach within FCA to analyze complex data such as ontology-based annotations of biomedical documents. In this paper, we propose a framework based on pattern structures for dealing with conceptual annotations which are made of sets of concepts represented within an $\mathcal{E} \mathcal{L}$ ontology. Then we propose a pattern structure providing a classification of biomedical documents according to their annotations and the semantic types of the concepts within the annotations. The resulting concept lattice can be used for analyzing and completing the original annotations.

This work shows that pattern structures are an efficient means for dealing with real-world and complex data. In the present case, more experiments remain to be done as well as a thorough study of the various pattern structures that can be associated to an annotation process depending on one or several ontologies.

\section{References}

[1] Franz Baader, Sebastian Brandt, and Carsten Lutz. Pushing the el envelope. In Leslie Pack Kaelbling and Alessandro Saffiotti, editors, IJCAI, pages 364-369. Professional Book Center, 2005.

[2] Franz Baader, Diego Calvanese, Deborah L. McGuinness, Daniele Nardi, and Peter F. Patel-Schneider, editors. The Description Logic Handbook: Theory, Implementation, and Applications. Cambridge University Press, 2003.

[3] Franz Baader, Ralf Küsters, and Ralf Molitor. Computing least common subsumers in description logics with existential restrictions. In IJCAI, pages 96-103, 1999.

[4] M. Barbut and B. Monjardet, editors. Ordres et classification: Algèbre et combinatoire (tome II). Hachette, Paris, 1970.

[5] Olivier Bodenreider. The unified medical language system (umls): integrating biomedical terminology. Nucleic Acids Research, 32(Database-Issue):267-270, 2004.

[6] Atul J. Butte. Viewpoint paper: Translational bioinformatics: Coming of age. JAMIA, 15(6):709-714, 2008.

[7] B. Ganter and S. O. Kuznetsov. Pattern Structures and Their Projections. In ICCS, volume 2120 of LNCS, pages 129-142. Springer, 2001.

[8] B. Ganter and R. Wille. Formal Concept Analysis. Springer, mathematical foundations edition, 1999.

[9] Clement Jonquet, Paea LePendu, Sean M. Falconer, Adrien Coulet, Natalya Fridman Noy, Mark A. Musen, and Nigam H. Shah. Ncbo resource index: Ontologybased search and mining of biomedical resources. J. Web Sem., 9(3):316-324, 2011.

[10] Mehdi Kaytoue, Sergei O. Kuznetsov, and Amedeo Napoli. Revisiting numerical pattern mining with formal concept analysis. In IJCAI, pages 1342-1347, 2011.

[11] Mehdi Kaytoue, Sergei O. Kuznetsov, Amedeo Napoli, and Sébastien Duplessis. Mining gene expression data with pattern structures in formal concept analysis. Inf. Sci., 181(10):1989-2001, 2011. 
[12] Craig Knox, Vivian Law, Timothy Jewison, Philip Liu, Son Ly, Alex Frolkis, Allison Pon, Kelly Banco, Christine Mak, Vanessa Neveu, Yannick Djoumbou, Roman Eisner, Anchi Guo, and David S. Wishart. Drugbank 3.0: a comprehensive resource for 'omics' research on drugs. Nucleic Acids Research, 39(DatabaseIssue):1035-1041, 2011.

[13] Sergei O. Kuznetsov. A fast algorithm for computing all intersections of objects in a finite semi-lattice. Automatic Documentation and Mathematical Linguistics, 27(5):400-412, 2004.

[14] Sergei O. Kuznetsov and Mikhail V. Samokhin. Learning closed sets of labeled graphs for chemical applications. In Stefan Kramer and Bernhard Pfahringer, editors, ILP, volume 3625 of Lecture Notes in Computer Science, pages 190-208. Springer, 2005.

[15] Alexa T. McCray. An upper level ontology for the biomedical domain. Comp. Funct. Genom., 4:80-84, 2003.

[16] Catia Pesquita, Daniel Faria, André O. Falcão, Phillip Lord, and Francisco M. Couto. Semantic similarity in biomedical ontologies. PLoS Computational Biology, 5(7), 2009.

[17] Daniel L. Rubin, Nigam Shah, and Natalya Fridman Noy. Biomedical ontologies: a functional perspective. Briefings in Bioinformatics, 9(1):75-90, 2008.

[18] Nicholas Sioutos, Sherri de Coronado, Margaret W. Haber, Frank W. Hartel, Wen-Ling Shaiu, and Lawrence W. Wright. Nci thesaurus: A semantic model integrating cancer-related clinical and molecular information. Journal of Biomedical Informatics, 40(1):30-43, 2007.

[19] Patricia L. Whetzel, Natalya Fridman Noy, Nigam H. Shah, Paul R. Alexander, Csongor Nyulas, Tania Tudorache, and Mark A. Musen. Bioportal: enhanced functionality via new web services from the national center for biomedical ontology to access and use ontologies in software applications. Nucleic Acids Research, 39(Web-Server-Issue):541-545, 2011. 